\title{
The relationship between glomerular IgG staining and poor prognostic findings in patients with IgA nephropathy: the data from TSN-GOLD working group
}

Kenan Turgutalp ${ }^{1 *}$, Egemen Cebeci ${ }^{2}$, Aydin Turkmen $^{3}$, Ulver Derici $^{4}$, Nurhan Seyahi $^{5}$, Necmi Eren $^{6}$, Fatih Dede ${ }^{7}$, Mustafa Gullulu ${ }^{8}$, Taner Basturk ${ }^{9}$, Gulizar Manga Sahin ${ }^{10}$, Murvet Yilmaz ${ }^{11}$, Savas Sipahi ${ }^{12}$, Garip Sahin ${ }^{13}$, Sena Ulu ${ }^{14}$, Erhan Tatar ${ }^{15}$, Ali Gundogdu ${ }^{16}$, Rumeyza Turan Kazancioglu ${ }^{17}$, Can Sevinc ${ }^{18}$, Ozkan Gungor ${ }^{19}$, Idris Sahin ${ }^{20}$, Sim Kutlay ${ }^{21}$, Ilhan Kurultak ${ }^{22}$, Zeki Aydin ${ }^{23}$, Bulent Altun ${ }^{24}$, Belda Dursun ${ }^{25}$, Zulfikar Yilmaz ${ }^{26}$, Ozcan Uzun ${ }^{27}$, Gultekin Suleymanlar ${ }^{28}$, Ferhan Candan ${ }^{29}$, Siren Sezer ${ }^{30}$, Derya Basak Tanburoglu ${ }^{31}$, Zerrin Bicik Bahcebasi ${ }^{32}$, Dilek Taymez ${ }^{33}$, Esra Akcali ${ }^{1}$, Deren Oygar ${ }^{34}$, Zulal Istemihan ${ }^{3}$, Simge Bardak ${ }^{34}$, Omer Faruk Akcay ${ }^{4}$, Mevlut Tamer Dincer ${ }^{5}$, Erkan Dervisoglu ${ }^{6}$, Ezgi Yenigun ${ }^{7}$, Kultigin Turkmen $^{35}$ and Savas Ozturk ${ }^{3}$

\begin{abstract}
Background: Galactose-deficient $\lg A 1$ (Gd-lgA1) has an increased tendency to form immunocomplexes with $\lg G$ in the serum, contributing to IgAN pathogenesis by accumulating in the glomerular mesangium. Several studies showed that glomerular IgG deposition in IgAN is an important cause of mesangial proliferation and glomerular damage. This study aims to determine the association of the positivity of $\lg G$ and the intensity of $\lg G$ staining with a poor renal prognosis.

Methods: A total of 943 IgAN patients were included in the study. Glomerular lgG staining negative and positive patients were compared using Oxford classification scores, histopathological evaluations, proteinuria, eGFR, albumin, blood pressures. IgG positive patients were classified as $(+),(++),(+++)$ based on their staining intensity, and the association with the prognostic criteria was also evaluated.
\end{abstract}

Results: $81 \%(n=764)$ of the patients were detected as IgG negative, while 19\% ( $n=179)$ were positive. Age, gender, body mass index, blood pressure, proteinuria, eGFR, uric acid values were similar in IgG positive and negative patients who underwent biopsy $(p>0.05)$. Intensity of glomerular lgG positivity was not found to be associated with diastolic and systolic blood pressure, urea, uric acid, age, eGFR, albumin, proteinuria ( $p>0.05$ for all, $r=-0.084, r=-0.102$, $r=-0.006, r=0.062, r=0.014, r=-0.044, r=-0.061, r=-0.066, r=0.150$, respectively). There was no difference for histopathological findings between $\lg G(+), \lg G(++), \lg G(+++)$ groups (for all, $p>0.05)$.

\footnotetext{
*Correspondence: k.turgutalp@hotmail.com

${ }^{1}$ Division of Nephrology, Department of Internal Medicine, School

of Medicine, Mersin University, 33079 Mersin, Turkey

Full list of author information is available at the end of the article
}

(c) The Author(s) 2021. Open Access This article is licensed under a Creative Commons Attribution 4.0 International License, which permits use, sharing, adaptation, distribution and reproduction in any medium or format, as long as you give appropriate credit to the original author(s) and the source, provide a link to the Creative Commons licence, and indicate if changes were made. The images or other third party material in this article are included in the article's Creative Commons licence, unless indicated otherwise in a credit line to the material. If material is not included in the article's Creative Commons licence and your intended use is not permitted by statutory regulation or exceeds the permitted use, you will need to obtain permission directly from the copyright holder. To view a copy of this licence, visit http://creativecommons.org/licenses/by/4.0/. The Creative Commons Public Domain Dedication waiver (http://creativeco mmons.org/publicdomain/zero/1.0/) applies to the data made available in this article, unless otherwise stated in a credit line to the data. 
Conclusion: Glomerular IgG negativity and positivity detected by routine IFM in IgAN patients is not associated with poor renal prognostic risk factors.

Keywords: IgA nephropathy, Glomerular lgG staining, Renal prognostic factors

\section{Background}

IgA nephropathy (IgAN) is the most common cause of primary glomerulonephritis throughout the world, including Turkey $[1,2]$. Although the prognosis may be difficult to predict, important risk factors for the progressive disease, including demographic, clinical and histopathological findings, have been identified. Male gender, amount of proteinuria, increased systolic and diastolic blood pressures, hyperuricemia, increased body mass index (BMI) and, increased age) have been defined for poor renal prognosis of IgAN [3]. The diagnosis requires a kidney biopsy, which often provides additional prognostic information: mesangial hypercellularity, endocapillary hypercellularity, segmental glomerulosclerosis, tubular atrophy/interstitial fibrosis and presence of crescents correlated with adverse renal outcomes [3].

Renal biopsies of IgAN patients are characterised by mesangial IgA dominant or codominant immunoglobulin deposits that may have co-deposition of IgG. Compared with healthy subjects, there is an increase in the proportion of Galactose-deficient IgA1(Gd-IgA1) in the serum. Gd-IgA1 has an increased tendency to form immunocomplexes with IgG in the serum, contributing to IgAN pathogenesis by accumulating in the glomerular mesangium. Several studies showed that glomerular IgG deposition in IgAN is an important cause of mesangial inflammation, which causes proliferation of the mesangial cells and glomerular damage. Some studies stated that IgG accumulation rather than IgA accumulation in mesangium was associated with poor renal outcome [1, 4, 5]. Animal models showed that the increased level of glomerular IgG accumulation is linked to the increased level of proteinuria [6]. Therefore, renal immunofluorescence microscopy (IFM) could also provide clinical or prognostic information. IFM fails to show IgG in up to $50-80 \%$ of kidney biopsies [1]; however, using more powerful methods, e.g. confocal microscopy, could help show glomerular IgG deposits. Thus, albeit not intensely stained or not detected with IFM, glomerular IgG may be associated with poor renal outcomes, such as severe staining cases.

The aim of this study is to determine whether IgG positivity and intensity of IgG staining detected by routine immunofluorescence microscopy (IFM) is associated with poor renal prognostic criteria.

\section{Methods}

\section{Study design and subjects}

This study is a retrospective, multicentre, cross-sectional study between May 2009-May 2019. The data of patients who underwent native kidney biopsy and diagnosed with 'primary glomerular disease' extracted from the 'Primary Glomerulonephritis Registry of Turkish Society of Nephrology' database by TSN-GOLD Working Group. Between 2009 and 2019, a total of 4399 patients from 47 nephrology centres all over Turkey were evaluated. Patients aged 16years or more with documented biopsy findings were included in the study. After excluding patients with missing kidney biopsy findings, the data of 3875 patients were evaluated. Among 3875 patients, a total of 943 patients had primary IgAN. The present study was approved by the Ethical Committee of the Istanbul Medical Faculty of Istanbul University.

\section{Renal histopathology}

The specimens were evaluated by the procedure under light microscopy and IFM. Light microscopic examination was carried out with paraffin-hidden tissue under hematoxylin-eosin, periodic acid-Schiff, aldehyde fuchsin orange $\mathrm{G}$, and periodic acid-silver methenamine stains. Snap-frozen 3- $\mu \mathrm{m}$-thick sections were used for routine IFM. Snap-frozen 3- $\mu \mathrm{m}$-thick sections were encountered with IgG antibodies beforehand. The semiquantitative force of IgG deposits in the mesangium, or glomerular capillary loops were evaluated by IFM in order to detect the presence of glomerular IgG. The deficiency of deposits in the mesangium or glomerular capillary loops were considered IgG negative. The presence of deposits in the mesangium or glomerular capillary loops were considered IgG positive. The IgG accumulation was scored as mild $[\operatorname{IgG}(+)]$, moderate $[\operatorname{IgG}(++)]$ and severe $[\operatorname{IgG}$ $(+++)]$.

The total number of glomeruli, global sclerotic glomeruli, and segmental sclerotic crescentic glomeruli were evaluated in the renal biopsies' materials. In addition, thickening of the basal membrane, mesangial proliferation, endocapillary proliferation and interstitial inflammation was evaluated. In addition, histopathologic findings such as mesangial hypercellularity, endocapillary hypercellularity, segmental sclerosis, tubular atrophy/ interstitial fibrosis belonging in the Oxford classification were also evaluated. The mesangial cellularity was scored as $0(<4$ mesangial cells/mesangial area), 1 (4-5 
cells), 2 (6-7 cells), and 3 ( $\geq 8$ cells) for each glomerulus. The mean score of all glomeruli was classified as M0 $(\leq 0.5)$ and M1 (>0.5). Endocapillary hypercellularity was categorised as either present (E1) or absent (E0). Similarly, segmental glomerulosclerosis was classified as either present (S1) or absent (S0). Tubular atrophy/interstitial fibrosis was classified as T0 (0-25\% of the cortical area), $\mathrm{T} 1$ (26-50\% of the cortical area), or T2 (>50\% of the cortical area) [7]. Interstitial fibrosis was defined as an increased extracellular matrix separating tubules in the cortical area. It is scored as percentage involvement, where $<1 \%$ was denoted as the absence of interstitial fibrosis (stage F0), with 1-5\% rounded to 5\% (stage F1, moderate interstitial fibrosis) and other values rounded to the nearest $10 \%$ (stage F2, severe interstitial fibrosis) [8].

\section{Data collection}

Age, gender, BMI, blood pressure and laboratory parameters including complete blood count, lipid profile, renal function tests, albumin, total protein, glucose, uric acid, calcium, alanine aminotransferase (ALT), complement 3 (C3), and complement 4 (C4) levels, 24-h proteinuria were collected and evaluated. Statistical differences of these parameters were evaluated between glomerular IgG negative and positive patients. These parameters were also evaluated in the subgroup analysis performed in IgG positive patients. The correlation between the intensity of glomerular IgG positive staining and age, urea, creatinine, eGFR, uric acid, albumin, urinary proteinuria, systolic blood pressure, diastolic blood pressure was evaluated.

The average of at least 2 systolic and diastolic blood pressure measurements was used. 24-h urine was collected to determine 24-h protein excretion. It was performed twice, and the average was calculated. eGFR was calculated by CKD-EPI formula [Chronic Kidney Disease Epidemiology Collaboration $)=141 \mathrm{X} \min (\mathrm{Scr} / \kappa, 1) \alpha \mathrm{X}$ $\max (\mathrm{Scr} / \kappa, 1)-1.209 \mathrm{X} 0.993 \mathrm{age} \times 1.018$ [female] X 1.159 [black]) [9]. Low-density lipoprotein cholesterol (LDL-C) level was assessed with Friedewald formula, which can be formulated as $\mathrm{LDL}=\mathrm{TC}-(\mathrm{HDL})-(\mathrm{TG} / 5)[10]$.

\section{Statistical analysis}

Statistical analyses were performed using Statistical Package for the Social Sciences (SPSS 22) software. Numerical variables that exhibited normal distribution were given as mean \pm standard deviation; the categorical variables as frequency and percentage. In the comparative analysis of demographic, laboratory and histopathological characteristics between IgG positive staining patients and IgG negative staining patients (Tables 1 and 3), independent groups were performed using the $\mathrm{t}$-test for parameters with normal distribution and the Chi-square test was used in the comparisons between categorical variables. ANOVA test was used to compare three groups according to the IgG staining intensity (Tables 2 and 4). For all statistical analyses, $p$-value $\leq 0.05$ was considered to be statistically significant.

\section{Results}

$81 \%(n=764)$ of the patients were detected as IgG negative, while $19 \%(n=179)$ were positive with IFM. However, subgroup analysis of glomerular IgG positive patients under IFM showed as follows: IgG $(+) n=131$, $\operatorname{IgG}(++) n=36, \operatorname{IgG}(+++) n=12$.

\section{Basic demographic and biochemical analyses of IgG negative and positive patients during the biopsy}

$63.7 \%$ of glomerular IgG positive patients and $62.7 \%$ of IgG negative patients were male $(p>0.05)$. While the mean eGFR of glomerular IgG positive patients was $69.7 \pm 37.9$, it was $66.5 \pm 36.8 \mathrm{ml} / \mathrm{min} / 1.73 \mathrm{~m}^{2}$ for $\mathrm{IgG}$ negative patients $(\mathrm{p}>0.05)$. The mean value of $24-\mathrm{h}$ urinary proteinuria was $3.20 \pm 0.48 \mathrm{~g}$ for glomerular IgG positive patients, whereas it was $3.24 \pm 0.45 \mathrm{~g}$ for IgG negative patients $(\mathrm{p}>0.05)$.

Demographic and biochemical findings of glomerular IgG positive and negative patients during the biopsy were shown in Table 1.

Intensity of glomerular IgG positivity was not associated with diastolic blood pressure, systolic blood pressure, urea, uric acid, age, eGFR, albumin, creatinine, 24-h urinary proteinuria or low serum C3 and C4 (for all $\mathrm{p}>0.05, \mathrm{r}=-0.084, \mathrm{r}=-0.102, \mathrm{r}=-0.006, \mathrm{r}=0.062$, $\mathrm{r}=0.014, \mathrm{r}=-0.044, \mathrm{r}=-0.061, \mathrm{r}=-0.066, \mathrm{r}=0.150$, $\mathrm{r}=-0.103, \mathrm{r}=0.012$, respectively).

\section{Basic demographic and biochemical analyses according to the intensity of glomerular lgG staining}

Basic demographic and biochemical analyses of the glomerular IgG positive patients were shown in Table 2 . There was no statistically significant difference between the groups in terms of the number of male patients, systolic blood pressure, diastolic blood pressure, BMI, uric acid, BUN, Creatinine, albumin, 24-h proteinuria or age.

\section{Histopathologic findings of IgG negative and positive patients during the biopsy}

Although endocapillary hypercellularity was significantly higher in patients with glomerular IgG positive compared to IgG negatives, there was no statistical difference $(p>0.05)$. T1 score of glomerular IgG negative patients was significantly higher compared to IgG positive patients $(p<0.05)$. Histopathologic findings and 
Table 1 Basic demographic and biochemical findings of lgG positive and IgG negative patients

\begin{tabular}{|c|c|c|c|}
\hline & $\begin{array}{l}\text { IgG positive patients } \\
\text { (n: 179) }\end{array}$ & $\begin{array}{l}\text { IgG negative patients } \\
\text { (n: 764) }\end{array}$ & $\mathbf{p}$ \\
\hline Age (years) & $38.5 \pm 13.1$ & $38.4 \pm 12.8$ & NS \\
\hline Gender (male) & $63.7 \%$ & $62.7 \%$ & NS \\
\hline BMI $\left(\mathrm{kg} / \mathrm{m}^{2}\right)$ & $26.4 \pm 5.1$ & $26.7 \pm 5.1$ & NS \\
\hline $\mathrm{SBP}(\mathrm{mmHg})$ & $130 \pm 22$ & $130 \pm 20$ & NS \\
\hline $\mathrm{DBP}(\mathrm{mmHg})$ & $82 \pm 13$ & $81 \pm 12$ & NS \\
\hline FBG (mg/dl) & $97 \pm 29$ & $95 \pm 23$ & NS \\
\hline BUN (mg/dl) & $26 \pm 22$ & $24 \pm 17$ & NS \\
\hline Creatinine (mg/dl) & $1.51 \pm 1.36$ & $1.56 \pm 1.25$ & NS \\
\hline eGFR (ml/min/1.73 $\left.\mathrm{m}^{2}\right)$ & $69.7 \pm 37.9$ & $66.5 \pm 36.8$ & NS \\
\hline Proteinuria (g/day) & $3.20 \pm 0.48$ & $3.24 \pm 0.45$ & NS \\
\hline Albumin (g/dl) & $3.9 \pm 0.6$ & $3.8 \pm 0.7$ & NS \\
\hline Uric acid (mg/dl) & $6.6 \pm 1.9$ & $6.6 \pm 2.0$ & NS \\
\hline Total protein (g/dl) & $7.6 \pm 0.9$ & $7.6 \pm 0.9$ & NS \\
\hline Total cholesterol (mg/dl) & $206 \pm 49$ & $215 \pm 62$ & NS \\
\hline LDL (mg/dl) & $129 \pm 41$ & $133 \pm 50$ & NS \\
\hline $\mathrm{HDL}(\mathrm{mg} / \mathrm{dl})$ & $46 \pm 17$ & $46 \pm 16$ & NS \\
\hline Triglycerides $(\mathrm{mg} / \mathrm{dl})^{*}$ & $163 \pm 99$ & $187 \pm 123$ & $<0.05$ \\
\hline Hemoglobin (g/dl) & $13 \pm 2$ & $13 \pm 2$ & NS \\
\hline Pyuria (leukocyte >5) & $17.7 \%$ & $20.6 \%$ & NS \\
\hline Haematuria (erythrocyte $>5$ ) & $70 \%$ & $72 \%$ & NS \\
\hline C3 low & $6.6 \%$ & $8.4 \%$ & NS \\
\hline C4 low & $0.9 \%$ & $0.9 \%$ & NS \\
\hline
\end{tabular}

Abbreviations: BMI Body mass index, SBP Systolic blood pressure, DBP Diastolic blood pressure, FBG fasting blood glucose, BUN Blood urea nitrogen, eGFR estimated glomerular filtration rate, $L D L$ Low density lipoprotein, $H D L$ High density lipoprotein, C Complement

Table 2 Basic demographic and biochemical analyses of $\operatorname{lgG}$ positive patient subgroup

\begin{tabular}{|c|c|c|c|c|}
\hline & $\begin{array}{l}\lg G(+) \\
(n: 131)\end{array}$ & $\begin{array}{l}\lg G(++) \\
(n: 36)\end{array}$ & $\begin{array}{l}\lg G(+++) \\
(\mathrm{n}: 12)\end{array}$ & $\mathbf{p}$ \\
\hline Age (years) & $38.2 \pm 12.8$ & $40.3 \pm 15.0$ & $36.5 \pm 11.2$ & NS \\
\hline Gender (male) & $63.4 \%$ & $63.9 \%$ & $66.7 \%$ & NS \\
\hline BMI $\left(\mathrm{kg} / \mathrm{m}^{2}\right)$ & $26.4 \pm 5.1$ & $26.1 \pm 4.6$ & $27.5 \pm 10.4$ & NS \\
\hline $\mathrm{SBP}(\mathrm{mmHg})$ & $131 \pm 23$ & $128 \pm 22$ & $119 \pm 15$ & NS \\
\hline $\mathrm{DBP}(\mathrm{mmHg})$ & $83 \pm 12$ & $80 \pm 14$ & $81 \pm 13$ & NS \\
\hline FBG(mg/dl) & $99 \pm 31$ & $93 \pm 22$ & $90 \pm 21$ & NS \\
\hline BUN (mg/dl) & $26 \pm 23$ & $26 \pm 20$ & $24 \pm 12$ & NS \\
\hline Creatinine (mg/dl) & $1.56 \pm 1.53$ & $1.39 \pm 0.69$ & $1: 32 \pm 0.34$ & NS \\
\hline eGFR $\left(\mathrm{ml} / \mathrm{min} / 1.73 \mathrm{~m}^{2}\right)$ & $70.4 \pm 39.0$ & $69.6 \pm 38.7$ & $61.6 \pm 16.7$ & NS \\
\hline Proteinuria (g/day) & $3.16 \pm 0.50$ & $3.27 \pm 0.38$ & $3.37 \pm 0.48$ & NS \\
\hline Albumin (g/dl) & $3.9 \pm 0.5$ & $3.9 \pm 0.9$ & $3.6 \pm 0.7$ & NS \\
\hline Uric acid (mg/dl) & $6.6 \pm 2.0$ & $7.6 \pm 1.8$ & $7.1 \pm 1.5$ & NS \\
\hline C3 low & $7.5 \%$ & $8 \%$ & $10 \%$ & NS \\
\hline C4 low & $5.5 \%$ & $5.2 \%$ & $6 \%$ & NS \\
\hline $\begin{array}{l}\text { Haematuria (erythro- } \\
\text { cytes }>5 \text { ) }\end{array}$ & $69.7 \%$ & $75 \%$ & $87.5 \%$ & NS \\
\hline
\end{tabular}

Abbreviations: BMI Body mass index, SBP Systolic blood pressure, DBP Diastolic blood pressure, $F B G$ fasting blood glucose, BUN Blood urea nitrogen, eGFR estimated glomerular filtration rate, C Complement statistically significant differences between glomerular IgG positive and negative patients were shown in Table 3.

\section{Histopathologic findings according to the intensity of glomerular lgG staining during the biopsy}

There was no difference for histopathological findings between IgG $(+)$, IgG $(++)$, IgG $(+++)$ groups (for all, $\mathrm{p}>0.05$ ). Histopathologic findings of the glomerular IgG positive patients' and their comparative analyses were shown in Table 4.

\section{Discussion}

Several studies described the relationship between glomerular IgG staining and poor prognostic markers in patients with IgA nephropathy. The difference between our study from these studies is that this study is a multicentre study with a large number of patients. To the best of our knowledge, our study is the first study that the intensity of glomerular IgG staining in IgAN patients was not related to poor prognostic factors and emphasises the importance of IgG positivity, as well as IgG negativity in IgAN patients.

In this retrospective study performed by the TSNGOLD Working Group, no difference was found between 
Table 3 Histopathologic findings of IgG positive and lgG negative patients

\begin{tabular}{|c|c|c|c|}
\hline & IgG positive patients ( $\mathrm{n}: 179$ ) & $\begin{array}{l}\text { IgG negative patients } \\
\text { (n: 764) }\end{array}$ & $\mathbf{p}$ \\
\hline Total glomerulus number & $18 \pm 10$ & $17 \pm 10$ & NS \\
\hline Global sclerotic glomerulus number & $4 \pm 4$ & $4 \pm 4$ & NS \\
\hline Segmental sclerotic glomerulus number & $2 \pm 3$ & $1 \pm 2$ & NS \\
\hline Cellular crescent number & $0 \pm 1$ & $0 \pm 1$ & NS \\
\hline Fibrocellular crescent number & $0 \pm 1$ & $0 \pm 2$ & NS \\
\hline Basal membrane thickening & $22.9 \%$ & $20.9 \%$ & NS \\
\hline Mesangial proliferation & $86.2 \%$ & $86.4 \%$ & NS \\
\hline Endocapillary proliferation & $24.2 \%$ & $18.3 \%$ & NS \\
\hline Interstitial inflammation & $72.0 \%$ & $74.7 \%$ & NS \\
\hline \multicolumn{4}{|l|}{ Tubular atrophy/interstitial fibrosis } \\
\hline T0 & $58.5 \%$ & $41.3 \%$ & $<0.05$ \\
\hline $\mathrm{T} 1$ & $35.3 \%$ & $44.7 \%$ & $<0.05$ \\
\hline $\mathrm{T} 2$ & $6.2 \%$ & $11 \%$ & NS \\
\hline \multicolumn{4}{|l|}{$\lg A$ staining } \\
\hline$(+)$ & $5.6 \%$ & $5.9 \%$ & NS \\
\hline$(++)$ & $27.5 \%$ & $33.5 \%$ & NS \\
\hline$(+++)$ & $68.6 \%$ & $60.5 \%$ & NS \\
\hline \multicolumn{4}{|l|}{ Mesangial hypercellularity } \\
\hline Mo & $23.5 \%$ & $22.2 \%$ & NS \\
\hline M1 & $76.5 \%$ & $78.8 \%$ & NS \\
\hline \multicolumn{4}{|l|}{ Endocapillary hypercellularity } \\
\hline E0 & $66.4 \%$ & $78.7 \%$ & NS \\
\hline $\mathrm{E} 1$ & $33.6 \%$ & $21.3 \%$ & NS \\
\hline \multicolumn{4}{|l|}{ Segmental glomerulosclerosis } \\
\hline so & $44.9 \%$ & $39.5 \%$ & NS \\
\hline S1 & $55.1 \%$ & $60.5 \%$ & NS \\
\hline
\end{tabular}

glomerular IgG negative and IgG positive IgAN patients for Oxford scores, biochemical, histopathological findings. In addition, no association between the intensity of glomerular IgG staining and poor renal prognostic factors such as 24-h urinary proteinuria, eGFR, creatinine, albumin, age, uric acid, systolic and diastolic blood pressures were found. Although the presence of glomerular in situ IgG/Gd-IgA1 immunocomplexes was shown in IgAN [11, 12], some experts suggest that routine IFM may be unsuccessful in detecting actually existing glomerular IgG $[13,14]$. There is some evidence that both deposition of IgG in glomerular mesangial space and in glomerular capillary loops are associated with poor prognostic factors. Although it has been reported that IgG accumulation in both mesangium and capillary loops is not different for poor renal prognostic factor [14], some studies have reported that IgG accumulation in capillary loops is highly related to poor renal prognosis than mesangial IgG accumulation [5]. As a result, glomerular IgG deposits in IgAN, independent from the location, may be associated with poor renal prognosis. In our study, we considered IgG positive if the presence of IgG deposits in the mesangium or glomerular capillary loops were positive. We did not find any difference between poor renal prognostic factors of patients with IgG negative and IgG positive. Moreover, we could not find a relationship between the intensity of the IgG positivity and poor renal prognostic factors in patients with IgG positive.

Galactose deficient IgA1 is formed in the circulation due to $\mathrm{O}$-glycosylation abnormality in the hinge region of IgA1s in patients with IgAN. Lymphocytes produce IgG against abnormal IgA1. IgG/Gd-IgA1 in situ immunocomplexes are formed, and these complexes accumulate in the mesangial or glomerular capillary loops in the glomeruli $[15,16]$. It is known that these in situ immunocomplexes are locally activating alternative and mannose-binding lectin pathways of the complement in IgAN [17]. IgG/Gd-IgA1 in situ immune complexes are cause the formation of the $\mathrm{C} 4 \mathrm{bC} 2 \mathrm{a}$ classical pathway by $\mathrm{C} 1$ fixation. As a result, $\mathrm{C} 3$ is converting to $\mathrm{C} 3 \mathrm{a}$ and $\mathrm{C} 3 \mathrm{~b}$. Activation of complements is causing the formation of 
Table 4 Histopathologic results of IgG positive patient subgroup

\begin{tabular}{|c|c|c|c|c|}
\hline & $\lg G(+)$ & $\lg G(++)$ & $\lg G(+++)$ & $\mathbf{p}$ \\
\hline Total glomerulus number & $18 \pm 10$ & $18 \pm 12$ & $22 \pm 10$ & NS \\
\hline Global sclerotic glomerulus number & $4 \pm 4$ & $4 \pm 5$ & $5 \pm 3$ & NS \\
\hline Segmental sclerotic glomerulus number & $2 \pm 3$ & $2 \pm 3$ & $2 \pm 2$ & NS \\
\hline Cellular crescent number & $1 \pm 2$ & $1 \pm 2$ & $2 \pm 4$ & NS \\
\hline Basal membrane thickening & $25.8 \%$ & $17.6 \%$ & $25.0 \%$ & NS \\
\hline Mesangial proliferation & $86.7 \%$ & $82.8 \%$ & $91.6 \%$ & NS \\
\hline Endocapillary proliferation & $22.8 \%$ & $24.1 \%$ & $42.8 \%$ & NS \\
\hline Interstitial inflammation & $71.3 \%$ & $71.4 \%$ & $81.8 \%$ & NS \\
\hline \multicolumn{5}{|l|}{ Tubular atrophy/interstitial fibrosis } \\
\hline T0 & $62.5 \%$ & $52.8 \%$ & $50.0 \%$ & NS \\
\hline $\mathrm{T} 1$ & $30.5 \%$ & $42.8 \%$ & $33.3 \%$ & NS \\
\hline $\mathrm{T} 2$ & $7 \%$ & $4.9 \%$ & $66.7 \%$ & NS \\
\hline \multicolumn{5}{|l|}{$\lg A$ staining } \\
\hline$(+)$ & $6.1 \%$ & $0 \%$ & $19.1 \%$ & NS \\
\hline$(++)$ & $28.7 \%$ & $27.7 \%$ & $9.2 \%$ & NS \\
\hline$(+++)$ & $65.2 \%$ & $62.3 \%$ & $72.7 \%$ & NS \\
\hline \multicolumn{5}{|l|}{ Mesangial hypercellularity } \\
\hline Mo & $26.7 \%$ & $14.2 \%$ & $16.6 \%$ & NS \\
\hline M1 & $73 \%$ & $85.8 \%$ & $\% 83.4 \%$ & NS \\
\hline \multicolumn{5}{|l|}{ Endocapillary hypercellularity } \\
\hline EO & 66.1 & 71.4 & $50.0 \%$ & NS \\
\hline E1 & $33.9 \%$ & $28.6 \%$ & $50.0 \%$ & NS \\
\hline \multicolumn{5}{|l|}{ Segmental glomerulosclerosis } \\
\hline So & $\% 45.0 \%$ & 38.0 & 66.6 & NS \\
\hline S1 & $\% 55.0 \%$ & 62.0 & 33.4 & NS \\
\hline
\end{tabular}

C3 convertase in the mesangium. C3 convertase cause C3 fragment and fragmented products of C3 [6].

According to this theory, C3 deposits in the mesangium causes severe histological lesions such as glomerulosclerosis or crescent formation. Moreover, the presence of IgG in the mesangial cells causes mesangiolysis and mesangial cellular activation in IgAN [18]. Shin et al. observed in their study that there was a positive correlation between IgG and C3 deposition, and glomerular IgG was associated with tubulointerstitial fibrosis in IgAN [4]. In our study, there was no correlation between glomerular IgG and glomerular C3 deposition. Chen et al. informed that low serum C3 levels were associated with poor prognostic factors in IgAN [15]. In our study, there was no correlation between low serum C3 levels and glomerular IgG positivity. Also, there was no difference in serum C3 level between IgG positive and negative patients.

IgA positivity alone is observed at a rate of approximately $25 \%$ by IFM in patients with IgAN [19]. On the other hand, IgG positivity has been occurred at a rate of $10-80 \%$ in IgAN [20]. IgG positivity in IgAN could aggravate glomerular inflammation and proteinuria [6].
It was reported in a Japanese cohort study that deposition of IgG in the capillary loops were increased proteinuria and associated with decreased renal functions [21]. In another study, it was reported that mesangial IgG deposition is associated with hypertension and decreased renal functions in IgAN patients [22]. A Korean study emphasised that the presence of glomerular IgG in IgAN patients is an independent risk factor for a poor renal outcome [4]. In a Japanese cohort study was reported that the intensity of IgG in the capillary loops was associated with a decrease in eGFR [23]. Likewise, IgG deposition in the mesangium and the capillary loops were associated with mortality and risk of renal replacement therapy in an Italian study [24]. However, in our study, there was no difference between IgG negative and IgG positive patients regarding poor prognostic criteria such as the amount of proteinuria, blood pressures, serum creatinine and eGFR levels. Moreover, no correlation was found between the intensity of IgG staining and the amount of proteinuria, serum creatinine, eGFR, or blood pressures. It is known that there is an association between Oxford classification and clinical renal outcome in IgAN [8]. 
However, glomerular IgG positivity was not evaluated in the Oxford classification. There are several studies in IgAN showing the relationship between IgG positivity detected by IFM and histological severity. Although Bellur et al. had shown the relationship between glomerular IgG positivity and endocapillary proliferation as well as mesangial cellularity, they could not find an association between IgG positivity and worse renal outcome [14]. In our study, there was no difference in Oxford scores of IgG positivity patients compared to IgG negative patients. In addition, as the intensity of IgG positivity increased, there was no difference in Oxford scores.

Rizk et al. examined kidney biopsies of IgG negative and positive in IgAN patients. They examined a total of 34 IgAN patients who were 14 IgG positive and 24 IgG negative with IFM. They included 6 primary membranous nephropathy and 8 lupus nephritis patients in their study as a control group [1]. IgG's were extracted from the renal biopsy materials of all the patients, and they observed the number of IgG's by ELISA. Confocal microscopy was used in six IgG positives and four IgG negative patients under IFM. Rizk et al. determined that the IgG autoantibodies in the kidney biopsy immunodeposits which were detected by both ELISA and IgG-IgA1 antigen were specific for Gd-IgA1. Even IgG negative by IFM, these autoantibodies were obtained when using either ELISA or IgG-IgA1 antigen by Rizk et al. In IgG negative biopsy samples, which were detected by using routine IFM, Rizk et al. demonstrated glomerular colocalisation of IgG and IgA using by confocal microscopy. These suggest that IgG positive biopsy samples have more Gd-IgA1-IgG immunocomplexes compared to IgG negatives.

Renal immune deposits in IgAN may be rich in IgG autoantibodies which are specific for Gd-IgA1, even if IgG's are not detected by routine IFM in IgAN kidneybiopsy specimens [1]. For these reasons, we may not have found a difference of poor renal prognostic risk factors between IgG negative and IgG positive patients in our study. Additionally, in our study, this may be one of the reasons why IgG positivity was not associated with poor renal prognostic risk factors. Even if IgG detected by routine IFM in IgAN glomeruli is mild, IgG may actually be severely positive.

\section{Limitations}

There are some limitations in our study: First, we didn't have follow-up data; therefore, we could not evaluate the association of IgG deposition with renal outcomes. Second, biopsy specimens were evaluated by different pathologists in each centre. Finally, IgG evaluation was not performed using confocal microscopy.

\section{Conclusions}

Glomerular IgG positivity detected by routine IFM in IgAN is not found to be associated with poor renal prognostic markers. Glomerular IgG negative and IgG positivity detected by routine IFM in IgAN patients is not associated with poor renal prognostic risk factors. Therefore, IgG evaluated by routine IFM might not be suitable for existing IgG severity detection.

\section{Abbreviations}

BMI: body mass index; IgAN: IgA nephropathy; C: Complement; CKD-EPI Formula: Chronic Kidney Disease Epidemiology Collaboration; eGFR: Estimated glomerular filtration rate; Gd-IgA1: Galactose deficient IgA1; IFM: Immunofluorescent microscopy; LM: Light microscopy; LDL-C: Low-density lipoprotein cholesterol.

\section{Acknowledgments \\ Not applicable.}

\section{Authors' contributions}

Conception, design and revised of the study (KT), Data Collection (KT, EC, AT, UD, NS, NE, FD, MG, TB, GMS, MY, SS, GS, SU, ET, AG, RTK, CS, OG, IS, SK, IK, ZA, $B A, B D, Z Y, O U, G S, F C, S S, D B T, Z B B, D T, E A, D O, Z I, S B, O F A, M T D, M E, E Y, K T, S O)$ statistical analysis (SO), interpretation of the data (SO, KT), article writing (KT), manuscript review and modification (KT). All authors read and approved the final version of the manuscript

\section{Funding}

Financial supports (establishing the registry database) have been obtained from the Turkish Society of Nephrology.

\section{Availability of data and materials}

The datasets used and/or analysed during the current study available from the corresponding author on reasonable request.

\section{Declarations}

\section{Ethics approval and consent to participate}

The written informed consent was obtained from all subjects before they participated in the study. This study was conducted according to the guidelines laid down by the Declaration of Helsinki. The present study was approved

by the Ethical Committee of the Istanbul Medical Faculty of Istanbul University.

\section{Consent for publication}

Not applicable.

\section{Competing interests}

The authors have no conflicts of interest to declare.

\section{Author details}

${ }^{1}$ Division of Nephrology, Department of Internal Medicine, School of Medicine, Mersin University, 33079 Mersin, Turkey. ${ }^{2}$ Department of Nephrology, Haseki Training and Research Hospital, Istanbul, Turkey. ${ }^{3}$ Department of Nephrology Istanbul University, Istanbul Faculty of Medicine, Istanbul, Turkey. ${ }^{4}$ Department of Nephrology, Gazi University Faculty of Medicine, Ankara, Turkey. ${ }^{5}$ Department of Nephrology, Istanbul University-Cerrahpasa, Cerrahpasa Faculty of Medicine, Istanbul, Turkey. ${ }^{6}$ Department of Nephrology Kocaeli University, Faculty of Medicine, Kocaeli, Turkey. ${ }^{7}$ Department of Nephrology, Health Sciences University, Ankara Numune Education and Research Hospital, Ankara, Turkey. ${ }^{8}$ Department of Medicine, Uludag University Faculty of Medicine, Bursa, Turkey. ${ }^{9}$ Department of Nephrology, Health Sciences University, Sislı Hamidiye Etfal Training and Research Hospital, Istanbul, Turkey. ${ }^{10}$ Department of Nephrology, Istanbul 2. Abdulhamid Han Training and Research Hospital, Istanbul, Turkey. ${ }^{11}$ Department of Nephrology, Health Sciences University, Bakirkoy Sadi Konuk Training and Research Hospital, Istanbul, Turkey. ${ }^{12}$ Department of Nephrology, Sakarya University 
Medical Faculty Education and Research Hospital, Istanbul, Turkey. ${ }^{13}$ Department of Nephrology, Eskisehir Osmangazi University, Faculty of Medicine, Eskisehir, Turkey. ${ }^{14}$ Department of Nephrology, Faculty of Medicine, Afyon Health Sciences University, Afyon, Turkey. ${ }^{15}$ Department of Nephrology, Health Sciences University, Izmir Bozyaka Training and Research Hospital, Izmir, Turkey. ${ }^{16}$ Department of Nephrology, Erciyes University, FAculty of Medicine, Kayseri, Turkey. ${ }^{17}$ Division of Nephrology, Bezmialem Vakif University, Faculty of Medicine, Istanbul, Turkey. ${ }^{18}$ Department of Nephrology, Atatürk University, School of Medicine, Erzurum, Turkey. ${ }^{19}$ Department of Nephrology, Kahramanmaras Sutcu Imam University, FAculty of Medicine, Kahramanmaraş, Turkey. ${ }^{20}$ Department of Nephrology, Inonu University Faculty of Medicine, Malatya, Turkey. ${ }^{21}$ Department of Nephrology, Ankara University, School of Medicine, Ankara, Turkey. ${ }^{22}$ Department of Nephrology, Trakya University, Faculty of Medicine, Edirne, Turkey. ${ }^{23}$ Department of Nephrology, Darica Farabi Training and Research Hospital, Kocaeli, Turkey. ${ }^{24}$ Department of Nephrology, Hacettepe University, Faculty of Medicine Ankara, Ankara, Turkey. ${ }^{25}$ Department of Nephrology, Pamukkale University, Scool of Medicine, Denizli, Turkey. ${ }^{26}$ Department of Nephrology Dicle University, School of Medicine, Diyarbakır, Turkey. ${ }^{27}$ Department of Nephrology Dokuz Eylül University, Faculty of Medicine, İmir, Turkey. ${ }^{28}$ Department of Nephrology Akdeniz University, School of Medicine, Antalya, Turkey. ${ }^{29}$ Department of Nephrology Cumhuriyet University, School of Medicine, Sivas, Turkey. ${ }^{30}$ Department of Nephrology Atilim University, School of Medicine, Medicana International Hospital, Ankara, Turkey. ${ }^{31}$ Department of Nephrology Cukurova University, School of Medicine, Adana, Turkey. ${ }^{32}$ Department of Nephrology, Kartal Lutfi Kirdar Training and Research Hospital, Istanbul, Turkey. ${ }^{33}$ Department of Nephrology, Kocaeli Government Hospital, Kocaeli, Turkey. ${ }^{34}$ Department of Nephrology, Lefkosa BND State Hospital, Lefkosa, Cyprus. ${ }^{35}$ Department of Internal Medicine, Division of Nephrology, Necmettin Erbakan University, Meram School of Medicine, Konya, Turkey.

Received: 3 May 2021 Accepted: 9 September 2021

Published online: 28 October 2021

\section{References}

1. Rizk DV, et al. Glomerular Immunodeposits of patients with IgA nephropathy are enriched for IgG autoantibodies specific for galactose-deficient IgA1. J Am Soc Nephrol. 2019;30(10):2017-26.

2. Sumnu, A. and A. Yildiz, Glomerülonefritlere genel bakış, in Primer glomerüler hastalıkların tanısı ve tedavisi: Türk Nefroloji Derneği Ulusal Uzlaşı Raporu., S. Öztürk, A. Sumnu, and E. Cebeci, Editors. 2019, BULUŞ Tasarım ve Matbaacılık Hizmetleri San. Tic.: Ankara. p. 27-39.

3. Rodrigues JC, Haas M, Reich HN. IgA nephropathy. Clin J Am Soc Nephrol. 2017:12(4):677-86.

4. Shin $\mathrm{DH}$, et al. Glomerular lgG deposition predicts renal outcome in patients with IgA nephropathy. Mod Pathol. 2016;29(7):743-52.

5. Alvarado AS, et al. Location of glomerular immune deposits, not codeposition of immunoglobulin G, influences definitive renal outcomes in immunoglobulin a nephropathy. Nephrol Dial Transplant. 2018;33(7):1168-75.
6. Van Dixhoorn MGA, et al. Combined glomerular deposition of polymeric rat IgA and IgG aggravates renal inflammation. Kidney Int. 2000;58(1):90-9.

7. Trimarchi H, et al. Oxford classification of IgA nephropathy 2016: an update from the IgA nephropathy classification working group. Kidney Int. 2017;91(5):1014-21.

8. Roberts ISD, et al. The Oxford classification of IgA nephropathy: pathology definitions, correlations, and reproducibility. Kidney Int. 2009;76(5):546-56.

9. Levey AS, et al. A new equation to estimate glomerular filtration rate. Ann Intern Med. 2009;150(9):604-12.

10. Friedewald WT, Levy RI, Fredrickson DS. Estimation of the concentration of low-density lipoprotein cholesterol in plasma, without use of the preparative ultracentrifuge. Clin Chem. 1972;18(6):499-502.

11. Hiki Y, et al. Mass spectrometry proves under-O-glycosylation of glomeruIar IgA1 in IgA nephropathy. Kidney Int. 2001;59(3):1077-85.

12. Allen $A C$, et al. Mesangial IgA1 in IgA nephropathy exhibits aberrant O-glycosylation: observations in three patients. Kidney Int. 2001;60(3):969-73.

13. Wada Y, et al. Clinical significance of IgG deposition in the glomerular mesangial area in patients with IgA nephropathy. Clin Exp Nephrol. 2013;17(1):73-82.

14. Bellur SS, et al. Immunostaining findings in IgA nephropathy: correlation with histology and clinical outcome in the Oxford classification patient cohort. Nephrol Dial Transplant. 2011;26(8):2533-6.

15. Chen $P$, et al. Plasma galactose-deficient IgA 1 and $C 3$ and CKD progression in IgA nephropathy. Clin J Am Soc Nephrol. 2019;14(10):1458-65.

16. Xu L-X, Zhao M-H. Aberrantly glycosylated serum IgA1 are closely associated with pathologic phenotypes of IgA nephropathy. Kidney Int. 2005;68(1):167-72.

17. Nakagawa $\mathrm{H}$, et al. Significance of glomerular deposition of $\mathrm{C} 3 \mathrm{C}$ and $\mathrm{C} 3 \mathrm{~d}$ in IgA nephropathy. Am J Nephrol. 2000;20(2):122-8.

18. Waldo FB. Role of IgA in IgA nephropathy. J Pediatr. 1990;1 16(5):78-85.

19. Rodicio JL. Idiopathic IgA nephropathy. Kidney Int. 1984;25(4):717-29.

20. Habib R, Niaudet P, Levy M. Schonlein-Henoch purpura nephritis and IgA nephropathy. In: Tisher C, Brenner B, editors. Renal Pathology with Clinical and Functional Correlations. Philadelphia, Pennsylvania, USA: Lippincott \& co; 1994. p. 472-23.

21. Yoshimura M, et al. Significance of IgA deposits on the glomerular capillary walls in IgA nephropathy. Am J Kidney Dis. 1987;9(5):404-9.

22. Nieuwhof $\mathrm{C}$, et al. Chronicity index and mesangial lgG deposition are risk factors for hypertension and renal failure in early IgA nephropathy. Am J Kidney Dis. 1998;31(6):962-70.

23. Kobayashi Y, et al. IgA nephropathy: prognostic significance of proteinuria and histological alterations. Nephron. 1983;34(3):146-53.

24. D'Amico G, et al. Prognostic indicators in idiopathic IgA mesangial nephropathy. Q J Med. 1986;59(228):363-78.

\section{Publisher's Note}

Springer Nature remains neutral with regard to jurisdictional claims in published maps and institutional affiliations.

\footnotetext{
Ready to submit your research? Choose BMC and benefit from:

- fast, convenient online submission

- thorough peer review by experienced researchers in your field

- rapid publication on acceptance

- support for research data, including large and complex data types

- gold Open Access which fosters wider collaboration and increased citations

- maximum visibility for your research: over $100 \mathrm{M}$ website views per year
}

At BMC, research is always in progress.

Learn more biomedcentral.com/submissions 